\title{
Myeloperoxidase-derived 2-chlorofatty acids contribute to human sepsis mortality via acute respiratory distress syndrome
}

\author{
Nuala J. Meyer, ${ }^{1,2}$ John P. Reilly, ${ }^{1,2}$ Rui Feng, ${ }^{3}$ Jason D. Christie, ${ }^{1,2,3}$ Stanley L. Hazen, ${ }^{4}$ \\ Carolyn J. Albert, ${ }^{5,6}$ Jacob D. Franke, ${ }^{5,6}$ Celine L. Hartman, ${ }^{5,6}$ Jane McHowat, ${ }^{6,7}$ and David A. Ford ${ }^{5,6}$ \\ 'Pulmonary, Allergy, and Critical Care Division, ${ }^{2}$ Center for Translational Lung Biology, and ${ }^{3}$ Center for Clinical Epidemiology \\ and Biostatistics, University of Pennsylvania Perelman School of Medicine, Philadelphia, Pennsylvania, USA. \\ ${ }^{4}$ Department of Cellular and Molecular Medicine, Center for Microbiome and Human Health, and Department of \\ Cardiovascular Medicine, Heart and Vascular Institute, Cleveland Clinic, Cleveland, Ohio, USA. ${ }^{5}$ Department of \\ Biochemistry and Molecular Biology, ${ }^{6}$ Center for Cardiovascular Research and 'Department of Pathology, Saint Louis \\ University School of Medicine, St. Louis, Missouri, USA.
}

\begin{abstract}
Sepsis-associated acute respiratory distress syndrome (ARDS) is characterized by neutrophilic inflammation and poor survival. Since neutrophil myeloperoxidase (MPO) activity leads to increased plasma 2-chlorofatty acid (2-CIFA) levels, we hypothesized that plasma concentrations of 2-CIFAs would associate with ARDS and mortality in subjects with sepsis. In sequential consenting patients with sepsis, free 2-CIFA levels were significantly associated with ARDS, and with 30-day mortality, for each log increase in free 2-chlorostearic acid. Plasma MPO was not associated with either ARDS or 30-day mortality but was correlated with 2-CIFA levels. Addition of plasma 2-CIFA levels to the APACHE III score improved prediction for ARDS. Plasma 2-CIFA levels correlated with plasma levels of angiopoietin-2, E selectin, and soluble thrombomodulin. Endothelial cells treated with 2-CIFA responded with increased adhesion molecule surface expression, increased angiopoietin-2 release, and dose-dependent endothelial permeability. Our results suggest that 2-CIFAs derived from neutrophil MPO-catalyzed oxidation contribute to pulmonary endothelial injury and have prognostic utility in sepsis-associated ARDS.
\end{abstract}

Conflict of interest: S.L. Hazen is named as a coinventor on patents held by the Cleveland Clinic relating to cardiovascular diagnostics and therapeutics, is a paid consultant for Procter \& Gamble, and has received research funds from Procter \& Gamble, Pfizer Inc., Roche Diagnostics, and Takeda. S.L. Hazen reports that he is eligible to receive royalty payments for inventions or discoveries related to cardiovascular diagnostics or therapeutics from Cleveland HeartLab, Esperion, and Frantz Biomarkers LLC. N.J. Meyer and J.D. Christie have received research funds from GlaxoSmithKline for work unrelated to the present manuscript. N.). Meyer has served on the advisory board for SOBI Inc.

Submitted: July 24, 2017 Accepted: November 1, 2017 Published: December 7, 2017

\section{Reference information:} JCI Insight. 2017;2(23):e96432. https://doi.org/10.1172/jci. insight.96432.

\section{Introduction}

Sepsis is a major cause of mortality in critically ill patients and is accompanied by development of a dysregulated host immune response to infection (1). Sepsis-induced organ failure is multifactorial and is believed to arise in part due to the interplay among pathogen, blood, and endothelial surface at the microcirculatory level, with the loss of microvascular endothelial barrier integrity and blood-endothelial interface dysfunction $(2,3)$. Bioactive lipids have received considerable attention as potential mediators of the pathophysiological sequelae of sepsis and inflammation (4-8). Bioactive lipids have also been implicated as potential mediators of the acute respiratory distress syndrome (ARDS), a common sepsis-induced organ failure with high observed mortality rates (9-11). Neutrophils and monocytes are major contributors to the generation of bioactive lipids. We sought to elucidate one potential mechanism of leukocyte-mediated damage during human sepsis by characterizing the plasma 2-chlorofatty acid (2-ClFA) profile and testing the clinical prognostic utility of plasma 2-ClFAs for prediction of ARDS.

Dysregulated balance between the antimicrobial host defense action of leukocytes and leukocyte-mediated tissue injury characterizes sepsis (12-14) and may have a key role in sepsis-associated organ failure (15-19). Autopsy specimens from human sepsis patients reveal neutrophil aggregation and infiltration of the kidney and lung, and the amount of neutrophil infiltration in ARDS patients correlates with lung dysfunction (15-19). Moreover, the gene expression signature of sepsis-associated ARDS is dominated by neutrophil-related genes (20). A major antimicrobial mechanism of neutrophil-associated phagosomes is the generation of reactive oxidant species $(13,14,21)$. Myeloperoxidase (MPO), the most abundant granule protein released from neutrophils 

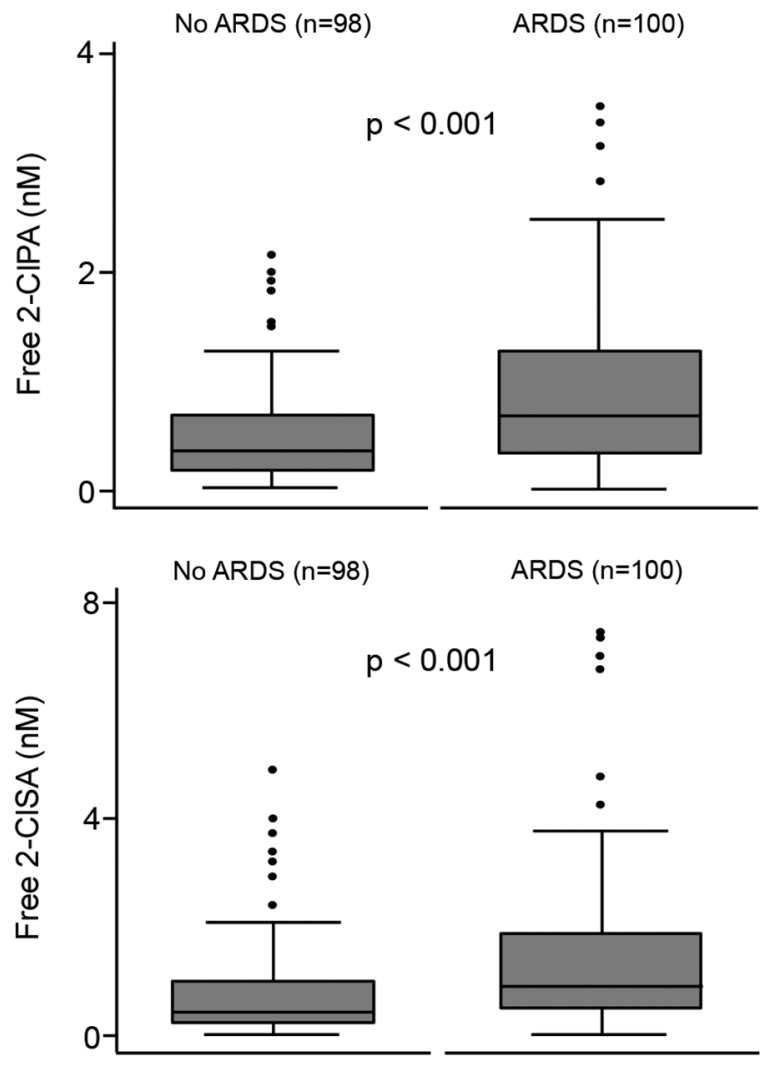

Figure 1. Septic patients with ARDS display higher day 0 plasma concentrations of free 2-CIFAs. Compared with subjects who never developed ARDS, those who developed ARDS within the first 6 days of sepsis have higher circulating levels of free 2-CIPA and free 2-CISA on the day of ICU admission (compared by the Wilcoxon rank-sum test). All values were included in the analyses; however, outliers higher than the $y$ axis are not shown. Box plots show median and 25th and 75th percentile, and whiskers show minima and maxima for each condition. For the ARDS group, 9 and 11 outliers had values above $4 \mathrm{nM}$ 2-CIPA or above $8 \mathrm{nM} 2$-CISA, respectively. In the non-ARDS group, there were 2 outliers with values above $8 \mathrm{nM}$ 2-CISA, but none above 4 nM 2-CIPA.

and monocytes, is detectable in human plasma and increased in sepsis compared with sterile inflammation or controls (22). MPO activity amplifies the oxidizing potential of neutrophil-produced hydrogen peroxide $\left(\mathrm{H}_{2} \mathrm{O}_{2}\right)$ by converting it to hypochlorous acid ( $\mathrm{HOCl})(23)$, a potent cytotoxic oxidizing agent. Plasmalogens represent a subclass of diradylglycerophospholipids enriched in vascular and pulmonary endothelial cells. The characteristic vinyl ether bond of plasmalogen phospholipids is highly susceptible to oxidation and anatomically positioned at the surface of the cell, as a major constituent within the plasmalemma. When exposed to $\mathrm{HOCl}$, an oxidant specifically generated by MPO, the plasmalogen oxidation product formed has been shown to be a corresponding free 2-chlorofatty aldehyde (2-CIFALD) $(24,25)$. 2-ClFALDs were among the first chlorinated lipids found in response to both neutrophil and monocyte activation (24-26). Plasmalogens are abundant phospholipids in the heart, lung, brain, kidney, and smooth muscle tissues as well as neutrophils, monocytes, and endothelial cells (27-34). The vinyl ether-linked aliphatic groups of plasmalogens are predominantly 16 and 18 carbons in length, which are released as 16 and 18 carbon 2-CIFALD molecular species following $\mathrm{HOCl}$ targeting (26). Subsequent investigations demonstrated that 2-ClFALDs are oxidized to 2-ClFAs, which can be further metabolized (35-37). Furthermore, $\mathrm{HOCl}$ produced by neutrophils has been shown to target plasmalogen in neighboring endothelial cells and extracellular lipoproteins, leading to 2-CIFALD production, and endothelial cells metabolize 2-CIFALD to 2-ClFA, which is released from endothelial cells $(25,26,37)$.

2-ClFALDs are short-lived in plasma samples, but our group has detected 2-ClFAs in plasma and demonstrated that plasma 2-ClFA levels are elevated in LPS-treated rats (36) and in Sendai virus-infected mice (35). These rodent studies suggest that chlorinated lipids are a component of the response to bacterial and viral infection. Leukocyte-mediated chlorinated lipid products have been shown to be biologically active species, capable of causing cardiac contractile dysfunction, NF- $\mathrm{kB}$ signaling in endothelial cells, endoplasmic reticulum stress, hydrogen peroxide production, and apoptosis (38-40). Accordingly, the present study was designed to investigate the role of 2-CIFAs in human sepsis, with a focus on ARDS given evidence for the contribution of neutrophils to ARDS $(20,41)$.

\section{Results}

To test the hypothesis that 2-ClFA levels are associated with outcomes in human sepsis, plasma samples were collected upon intensive care unit (ICU) admission (day 0) from 198 sequential subjects enrolled in the Molecular Epidemiology of $\underline{S}$ epsis in the ICU (MESSI) cohort (see Supplemental Table 1 for MESSI study population characteristics; supplemental material available online with this article; https:// doi.org/10.1172/jci.insight.96432DS1). Informed consent was obtained from patients or their proxies as previously described (42). Plasma was examined for two molecular species of both free (unesterified) 2-ClFA and total (esterified + unesterified) 2-ClFA content, 2-chloropalmitic acid (2-ClPA), and 2-chlorostearic acid (2-ClSA) by stable isotope dilution tandem mass spectrometry. These molecular species were chosen because they represent the major aliphatic groups derived from plasmalogens in mammalian tissues $(43,44)$. Additionally, free and total 2-ClFA were determined to assess short and long half-life forms of 2-ClFA, respectively (36). Admission plasma levels of both free and total 2-ClFAs were higher in subjects who developed ARDS (median 2-CISA $1.12 \mathrm{nM}$ [interquartile range $\{\mathrm{IQR}\}$ 0.48-2.54 nM compared with median $0.43 \mathrm{nM}$ [IQR $0.22-0.98 \mathrm{nM}$ ] for non-ARDS, $P<0.001$ ) or who died within 
Table 1. Day O plasma 2-CIFA concentrations associate with ARDS

\begin{tabular}{ccc}
\hline & Adjusted odds ratio for ARDS (95\% CI) & P value \\
Log(free 2-CIPA) nM & $1.62(1.25,2.09)$ & $<0.001$ \\
Log(free 2-CISA) nM & $1.82(1.41,2.37)$ & $<0.001$ \\
$\log ($ total 2-CIPA) nM & $1.82(1.32,2.52)$ & $<0.001$ \\
$\log ($ total 2-CISA) nM & $1.78(1.31,2.43)$ & $<0.001$ \\
$\log (\mathrm{MPO}) \mathrm{pM}(n=110)^{\mathrm{A}}$ & $1.28(0.89,1.84)$ & 0.19
\end{tabular}

The odds for ARDS increase significantly among 198 subjects with sepsis for each log increase in free or total 2-CIPA or 2-CISA, independent of APACHE III score, age, sex, European descent, or pulmonary source of infection. In contrast, increasing MPO did not associate with ARDS in the subset $\left(n=110^{A}\right)$ with MPO measured. Associations remained unchanged when adjusting for absolute neutrophil count.

30 days compared with sepsis survivors $(P=0.0049)$ (Figure 1 and Supplemental Table 2). For each $\log$ increase in free 2-ClFA, the risk for ARDS (Table 1) and mortality (Table 2) significantly increased, and these associations remained robust following adjustment for the Acute Physiology and Chronic Health Evaluation (APACHE) III score, race, age, or sex. The APACHE III score is a validated score to adjust for baseline differences in expected mortality in critical illness (45). It is calculated based on a combination of clinical variables reflecting physiologic derangement, organ injury, age, and chronic health comorbidities that influence survival. Adjustment for absolute neutrophil count had no effect on the association between 2-ClFA concentration and ARDS or mortality. Because ARDS strongly influences sepsis mortality in this population, with odds ratio (OR) 4.7 (3.61-6.25), $P=0.0001$, we used statistical mediation analysis to explain the portion of the 2-ClFA-mortality association that was mediated by increased ARDS risk. For this analysis, ARDS risk was tested as the mediator variable between higher 2-ClFA concentration and mortality risk. As shown in Table 3, the mediation effect of ARDS on mortality was highly significant for both species of 2-ClFA. The development of ARDS accounted for $41 \%-51 \%$ of the mortality risk of higher 2-ClFA levels.

To test whether admission plasma concentrations of 2-ClFA had incremental value for predicting ARDS among septic subjects, we calculated receiver operating characteristic (ROC) curves and assessed the area under the ROC curve (AUROC) for predicting ARDS based on the APACHE III score alone or in combination with plasma 2-ClFA concentrations (Figure 2). The addition of free 2-ClSA levels to APACHE III improved the AUROC from 0.66 (95\% CI $0.59,0.74)$ to 0.76 (95\% CI $0.69,0.82)$, a significant improvement $(P=0.0042)$. Using the 50th percentile of free 2-CISA as a threshold, the net reclassification index for ARDS was 0.17 compared with APACHE III score alone $(P=0.026)$, and the integrated discrimination improvement (IDI) was $11 \%(P<0.001)$. Results were similar for the predictive utility of free 2-ClPA concentrations compared with APACHE score alone. Because 62 of the 100 subjects with ARDS developed ARDS on the same day as ICU admission (and thus the same day as blood draw), we repeated the analyses excluding subjects whose ARDS was present on day 0 and still observed a significant improvement in AUROC $(P=0.0030)$ and in IDI $(9.7 \%, P=0.001)$. Thus, we concluded that circulating levels of free 2-CIFA have predictive utility for ARDS independent of severity of illness.

Plasma 2-ClFA likely increases during sepsis as a result of systemic activation of neutrophils in response to sepsis. Almost $17 \%$ of the MESSI subjects were neutropenic on admission to the ICU secondary to prior

Table 2. Day 0 plasma 2-CIFA levels associate with sepsis mortality

\begin{tabular}{|c|c|c|}
\hline & Adjusted odds ratio for death at $30 \mathrm{~d}(95 \% \mathrm{Cl})$ & $P$ value \\
\hline Log(free 2-CIPA) nM & $1.34(1.07,1.68)$ & 0.011 \\
\hline Log(free 2-CISA) nM & $1.45(1.16,1.83)$ & 0.001 \\
\hline Log(total 2-CISA) nM & $1.31(1.00,1.71)$ & 0.048 \\
\hline $\log (\mathrm{MPO}) \mathrm{pM}\left(n=110^{\mathrm{A}}\right)$ & $1.00(0.68,1.47)$ & 0.99 \\
\hline
\end{tabular}

Association in 198 septic subjects was tested by logistic regression, adjusting for APACHE III score, age, sex, and race. In the subgroup with MPO tested ( $\left.n=110^{A}\right)$, there was no observable association between MPO concentration and mortality. Associations remained unchanged when adjusting for absolute neutrophil count. 
Table 3. Increased ARDS risk explains a significant portion of the association between plasma 2-CIFA concentrations and mortality

\begin{tabular}{cccc}
\hline & Adjusted odds ratio for mortality $\mathbf{( 9 5 \%} \mathrm{Cl})$ & P value & Proportion mediated by ARDS \\
Free 2-CIPA & $1.06(1.01,1.10)$ & 0.02 & $50.7 \%$ \\
Total effect & $1.03(1.01,1.05)$ & $<0.01$ & 0.23 \\
Mediation effect & $1.03(0.98,1.07)$ & & \\
Direct effect & $1.08(1.03,1.12)$ & $<0.01$ & $<0.01$ \\
Free 2-CISA & $1.03(1.01,1.05)$ & 0.07 & $41.6 \%$ \\
Total effect & $1.04(0.99,1.09)$ &
\end{tabular}

By mediation analysis, $40 \%-51 \%$ of mortality risk observed for free 2-CIFA concentrations is mediated through ARDS. Each effect shown reflects the average value of 1,000 iterations. The total effect model included the covariates $\log (2$-CIFA), ARDS, ARDS $\times \log (2$-CIFA) interaction term, age, APACHE III score, sex, and race. Results are shown separately for each 2-CIFA moiety, 2-CIPA and 2-CISA.

receipt of cytotoxic chemotherapy. Plasma 2-ClFA levels for both 2-ClPA and 2-ClSA seem to reflect the activity of circulating neutrophils, since plasma 2-ClFA levels were reduced in septic neutropenic subjects (Table 4). We also tested total and free plasma 2-ClFA concentrations in a small number of healthy volunteers $(n=4)$ and observed significantly lower plasma concentrations in nonsepsis controls compared with sepsis survivors and sepsis nonsurvivors: nonparametric test of trend, $P=0.007$ for total 2-ClPA; $P=0.003$ for free 2-ClPA; $P=0.001$ for free 2-ClSA (Supplemental Table 3). In a subgroup analysis of 110 of the subjects with sufficient plasma volume to measure MPO (described in Supplemental Table 4), we observed a strong correlation between plasma MPO levels and free 2-ClFAs and a modest correlation between MPO and total 2-ClFA levels (Spearman rank correlation, total 2-ClPA: rho 0.385, $P<0.001$; free 2-ClPA: rho 0.402, $P<0.001$; total 2-ClSA: rho 0.497, $P<0.001$; and free 2-CISA: rho 0.535, $P<0.001)$. Neutropenic subjects had significantly lower plasma levels of MPO $(P<0.001)$. However, whereas 2-ClFA levels were associated with ARDS and mortality (Tables 1 and 2), MPO levels for these 110 subjects were not significantly associated with ARDS $(P=0.19$, Table 1$)$ or mortality $(P=0.99$, Table 2$)$. Among the small subgroup with neutropenia $(n=31$ ), plasma 2-ClFA concentrations did not differ by ARDS status and were very similar to control subjects without sepsis (Supplemental Table 5). Plasma 2-ClFA concentrations did not distinguish Gram-negative bacteria sepsis from non-Gram-negative bacteria sepsis (Supplemental Table 6).

Multiorgan failure associated with sepsis is mediated by microcirculatory collapse, including dysfunction at the blood-endothelial interface where neutrophils interact with endothelial cells leading to injury. We measured plasma concentrations of angiopoietin-2 (Ang-2), vWF, E selectin, soluble thrombomodulin (sTM), and soluble ICAM-1 (sICAM-1) in MESSI subjects to determine the degree of correlation between markers of endothelial activation and plasma 2-ClFA concentrations. Several soluble factors indicating blood-endothelial interface dysfunction, including Ang-2, E selectin, and sTM, were correlated with 2-ClPA or 2-ClSA, though the degree of correlation was very weak to weak (rho 0.16-0.27) (Table 5). In contrast, plasma levels of vWF and sICAM-1 did not correlate with 2-ClFA levels in the MESSI cohort.

To provide better insights into whether the observed association between 2-ClFA and endothelial dysfunction may be mechanistically linked, human lung microvascular endothelial cells (HMVEC-L) were exposed to pathophysiologically relevant levels $(10 \mu \mathrm{M})$ of 2-ClPA and indicators of endothelial function were examined. Previous studies have shown local concentrations of 2-ClPA produced by

Table 4. Total and free plasma 2-CIFA molecular species levels are significantly lower in neutropenic subjects with sepsis

\begin{tabular}{|c|c|c|c|}
\hline & Neutropenic $(n=33)$ & Nonneutropenic $(n=164)$ & $P$ value \\
\hline Total 2-CIPA nM & $0.77(0.31,1.67)$ & $1.85(0.95,3.06)$ & $<0.001$ \\
\hline Free 2-CIPA nM & $0.31(0.16,0.61)$ & $0.60(0.28,1.19)$ & 0.005 \\
\hline Total 2-CISA nM & $0.75(0.34,2.18)$ & $2.68(1.26,4.61)$ & $<0.001$ \\
\hline Free 2-CISA nM & $0.24(0.06,0.70)$ & $0.76(0.37,2.91)$ & $<0.001$ \\
\hline MPO pM & $315(175,545)$ & $1519(871,2794)$ & $<0.001$ \\
\hline
\end{tabular}


Table 5. Plasma concentrations of 2-CIFA correlate with Ang-2, E selectin, and soluble thrombomodulin

\begin{tabular}{lccccc}
\hline & Ang-2 (pg/ml) & vWF (\% control) & E selectin (ng/ml) & sThrombomodulin (ng/ml) & sICAM-1 (ng/ml) \\
Free 2-CIPA & $0.22, P=0.0056^{\mathrm{A}}$ & $0.10, P=0.1876$ & $0.16, P=0.0403^{\mathrm{A}}$ & $0.27, P=0.0008^{\mathrm{A}}$ & $0.08, P=0.2974$ \\
Free 2-CISA & $0.12, P=0.1210$ & $0.06, P=0.4230$ & $0.24, P=0.0020^{\mathrm{A}}$ & $0.10, P=0.2143$ & $0.10, P=0.1968$
\end{tabular}

Spearman rank correlation (rho) and $P$ values between plasma endothelial markers and plasma-free 2-CIFA molecular species. ${ }^{A}$ Significant correlation. sThrombomodulin, soluble thrombomodulin; sICAM-1, soluble ICAM-1.

neutrophils and monocytes to be in the $10-30 \mu \mathrm{M}$ range $(25,35,40)$, whereas, in septic plasma, we observed concentrations of $0.5-100 \mathrm{nM}$. It is likely that systemic levels of 2-ClPA approaching 100 $\mathrm{nM}$ represent much higher levels of 2-ClPA produced at sites of inflammation and approach those levels found in activated neutrophils and monocytes. 2-ClPA was shown to elicit loss of endothelial permeability barrier (Figure 3A) but not alterations in cell metabolism, as ascertained by MTT assay (Supplemental Figure 1). In comparison, the nonchlorinated fatty acid precursor, palmitic acid (PA), had no effect on the HMVEC-L permeability barrier. Further studies shown in Figure 3B demonstrate a dose-dependent loss of human pulmonary microvascular resistance in response to 2-ClPA, with significant loss of permeability barrier at concentrations as low as $100 \mathrm{nM}(0.1 \mu \mathrm{M})$. Since Ang-2 associated with 2-ClFA in septic subjects and Ang-2 mediates endothelial barrier dysfunction, further investigations revealed the release of Ang-2 from human pulmonary microvascular endothelial cells treated with $10 \mu \mathrm{M}$ 2-ClPA (Figure 3C). In comparison, PA did not cause endothelial Ang-2 release. Moreover, in comparison to the known Weibel-Palade body stimulant, phorbol myristate acetate (46), 2-CIPA was a potent stimulator of Ang-2 release. 2-ClPA, and not PA, also led to increased adhesion of neutrophils (Figure 3D) and platelets (Figure 3E) to HMVEC-L. Exposure to 2-ClPA but not PA also led to enhanced surface expression of $\mathrm{P}$ selectin, E selectin, ICAM-1, and VCAM-1 (Figure 3F) in HMVEC-L. Lung endothelium may be particularly at risk to 2-ClFA-mediated injury, as human renal glomerular microvascular endothelial cells manifested a less pronounced fall in endothelial resistance compared with HMVEC-L and did not increase membrane surface expression of P selectin, E selectin, ICAM-1, or VCAM-1 in response to 2-ClPA (Supplemental Figure 2).

\section{Discussion}

The chlorinated lipids, 2-ClFALD and 2-ClFA, are produced as a result of plasmalogen oxidation during neutrophil activation and exposure to the MPO-generated oxidant, $\mathrm{HOCl}$. 2-ClFA has been shown to be present as a relatively stable chlorinated lipid produced as a result of inflammation, including endotoxemia

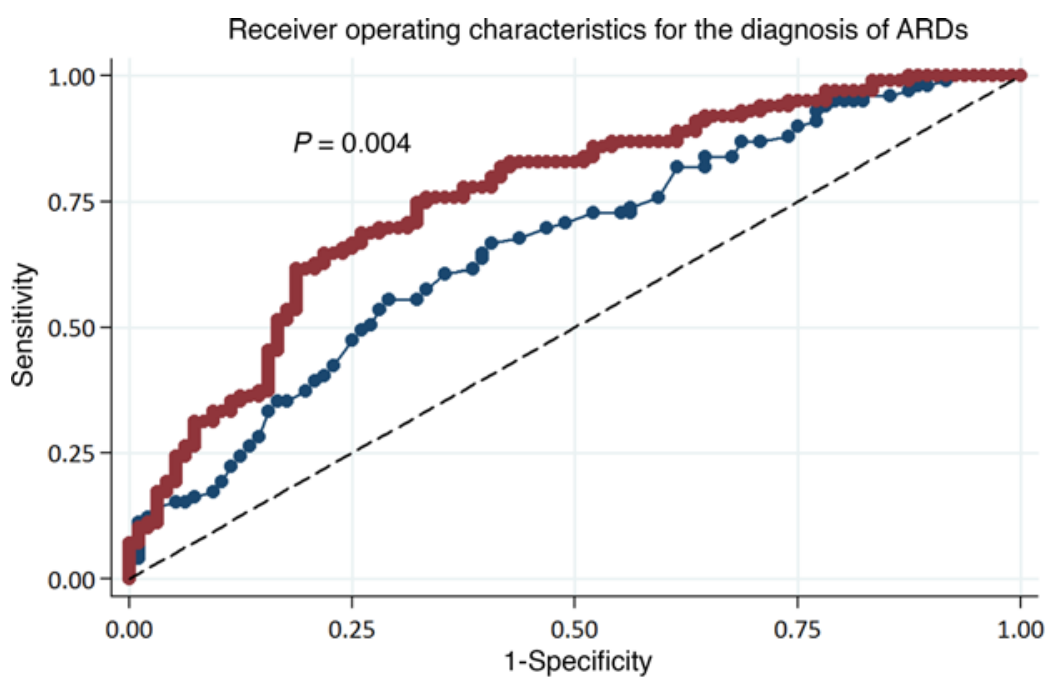

$\longrightarrow$ APACHE III ROC area: $0.66 \longrightarrow$ APACHE III+2-CISA ROC area: 0.76 ---- Reference
Figure 2. Neutrophil-derived plasma 2-CISA levels improve ARDS prediction. Areas under the receiver operating curve (ROC) for ARDS diagnosis were compared between a logistic regression model predicting ARDS based on APACHE III score alone, which encompasses illness severity and comorbidities contributing to intensive care unit mortality, and a model predicting ARDS based on APACHE III score and log-transformed free plasma 2-CISA concentrations. The improvement in area under the ROC (AUROC) from 0.66 to 0.76 is statistically significant $\left(P=0.0042\right.$ by $\chi^{2}$ test). 

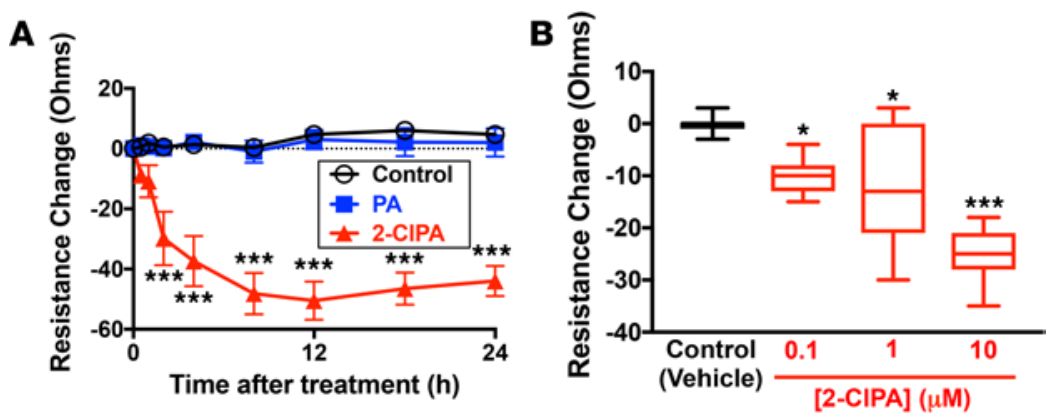

C
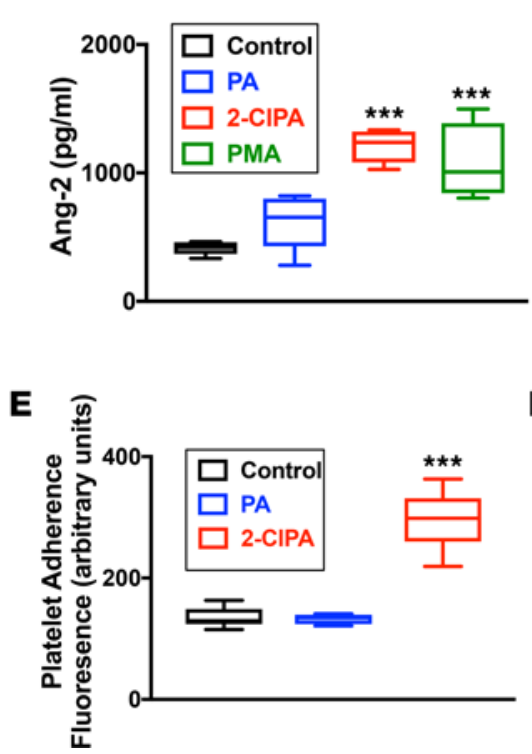
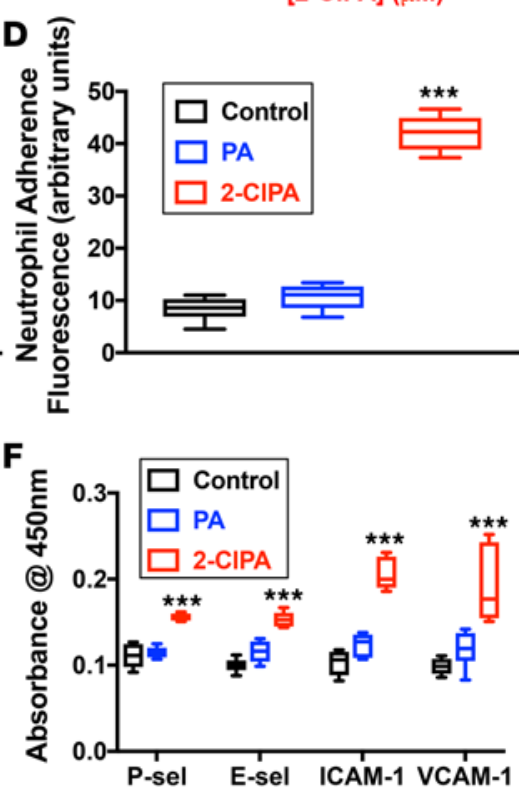

Figure 3. 2-CIPA induces Weibel-Palade body mobilization, leukocyte and platelet adherence, and permeability leakiness in human pulmonary microvascular endothelial cells. In comparison to PA, exogenously added 2-CIPA increases human pulmonary microvascular endothelial cell barrier permeability (A, $n=6$ for each data point; mean \pm SEM) (ANOVA with Tukey's post-hoc test), Ang-2 release $(C, n=5)$ (ANOVA with Tukey's post-hoc test), neutrophil adherence ( $\mathbf{D}, n=10$ ) (ANOVA with Tukey's post-hoc test), platelet adherence (E, $n=6$ ) (ANOVA with Tukey's post-hoc test), and surface adhesion molecule expression $(\mathbf{F}, n=8)$ (ANOVA with Tukey's post-hoc test). For $\mathbf{A}$ and $\mathbf{C}-\mathbf{F}$ the lipid addition was at $10 \mu \mathrm{M}$. (B) Concentration dependence of 2-CIPA (0.1-10 $\mu \mathrm{M}$ 2-CIPA) elicited endothelial permeability barrier disruption $(n=6)$ (ANOVA with Dunnett's post-hoc test). ${ }^{*} P<0.05$ and ${ }^{* *} P<0.001$ when compared with untreated controls. Box plots show median and 25 th and 75 th percentile, and whiskers show minima and maxima for each condition.

and Sendai virus exposure $(35,36)$. Additionally, lung 2-ClFA is elevated in mice exposed to chlorine gas, and intranasal administration of 2-ClFA resulted in increased protein and macrophage levels in bronchoalveolar fluid (47). The reported results demonstrate for the first time to our knowledge that systemic levels of 2-ClFA in sequential septic patients are associated with risk for ARDS and 30-day mortality. Moreover, plasma 2-CIFA concentrations correlate with clinical markers of endothelial dysfunction/injury in humans, and pathophysiologically relevant levels of 2-ClFA promote endothelial damage in microvascular endothelial cells in vitro. Taken together, the present findings indicate strong evidence for plasma 2-ClFA levels as a predictor of sepsis-associated ARDS, likely through effects on pulmonary microvasculature. Two molecular species of 2-ClFA have been assessed, 2-chloropalmitic acid and 2-chlorostearic acid. These species are derived from the saturated aliphatic groups linked to the glycerol backbone of plasmalogens by a vinyl ether bond. The initial chlorinated lipid product from HOCl-targeting plasmalogens is the short-lived 2-CIFALD, which is then oxidized into the free 2-ClFA species. Thus, free 2-ClFA is acutely produced during neutrophil activation, and over time some of the free 2-ClFA is stored in esterified pools, which comprise in part total 2-ClFA levels assessed in these studies. Esterified pools of 2-ClFA may in part reflect long-term storage of 2-ClFA, which may precede sepsis. We observed robust associations between ARDS and both free and total 2-ClFA species (Table 1). Comparing plasma 2-ClPA to 2-ClSA, these moieties manifested a similar degree of association with sepsis mortality and ARDS. However, because the molecular species of plasmalogens may vary between target organs, it is possible that 2-CISA and 2-CIPA levels may vary dependent on the target organ infiltrated with neutrophils. This should be considered for future investigations.

Although plasma MPO levels increase in humans with sepsis, indicative of systemic activation of neutrophils (48), MPO has not been shown to predict outcomes in septic patients. Consistent with prior literature (22), we did not observe an association between plasma MPO concentrations and outcomes in the MESSI cohort of sepsis patients. In contrast, plasma concentrations of 2-ClFAs, the stable oxidation products of MPO activity, associated with both ARDS and 30-day mortality, independent of neutrophil count. We posit that MPO is involved in the pathophysiology leading to organ damage, and its catalytic activity leads to the production of sta- 
ble metabolites with tighter associations with adverse outcomes. Taken together, these data are consistent with neutrophil activation as a rational mechanism for producing the elevated plasma 2-ClFA levels during sepsis.

The association of plasma 2-CIFA levels with ARDS, as well as with plasma Ang-2 levels, led to further investigations of the direct role of free 2-ClFA on pulmonary microvascular endothelial function. Lung microvascular endothelial cells were exposed to pathophysiologically plausible levels of 2-ClPA, which led to mobilization of Weibel-Palade bodies, resulting in the release of Ang-2 and the surface expression of $\mathrm{P}$ selectin and E selectin. In addition to Ang-2 release, 2-ClPA exposure caused permeability barrier dysfunction. Previous studies have shown that plasma Ang-2 levels are increased in human sepsis and have suggested that Ang-2 may contribute to ARDS through its tendency to deactivate Tie-2 receptor function, a key regulator of endothelial permeability (49). In addition to surface expression of P and E selectins, ICAM-1 and VCAM-1 were increased in 2-CIPA-treated endothelial cells. Accompanying the surface expression of adhesion molecules in response to 2-ClPA was an increased adherence of neutrophils and platelets to endothelial cells. Importantly, the changes elicited by 2-ClPA on endothelial permeability, Ang-2 release, surface adhesion molecule expression, and the adherence of neutrophils and platelets were not observed in cells treated with the corresponding nonchlorinated fatty acid, PA. The mechanisms responsible for the changes elicited specifically by 2-ClFA merit further investigation.

As evidenced by the more pronounced barrier dysfunction and the upregulation of markers of endothelial activation in HMVEC-L but not kidney endothelium in response to 2-ClFA, the lung may be particularly vulnerable to MPO-driven injury. The blood and lung fluid cytokine profile of ARDS is dominated by neutrophil chemoattractants IL-8 and G-CSF (50-53), and neutrophils may sequester in the lung microvasculature (54). Although patients with neutropenia do develop ARDS, it may be that neutropenic ARDS is mechanistically distinct from ARDS in subjects with neutrophils $(42,55)$, just as some animal models of lung injury are neutrophil dependent (56). In our underpowered subgroup of neutropenic subjects, those with ARDS had plasma 2-CIFA concentrations that were not different from non-ARDS subjects and that resembled concentrations observed in healthy controls. Thus, we hypothesize that the mechanism underlying ARDS in neutropenic subjects may be driven by direct epithelial and/or endothelial injury that is not chlorolipid dependent. In large clinical trials of ARDS, a hyperinflammatory subphenotype characterized by high circulating levels of IL-8, high IL-6, and Ang-2 has been described that associates with higher mortality and a differential response to ventilator and fluid management $(57,58)$. Because 2-CIFA may have additional specificity to identify neutrophil-mediated lung injury, these markers may have utility as potential enrichment factors for trials examining antiinflammatory $(59,60)$ or antipermeability treatments in ARDS (61).

Our study had several limitations. Our clinical cohort is an ideal design to test association between clinical and biological variation and sepsis-induced organ failure and outcome; however, the observational human portion of our study was not designed to prove causality. Nonetheless, the complementary in vitro data demonstrate that 2-CIFAs are sufficient to incite significant lung microvasculature activation and permeability, such as occurs during sepsis-associated ARDS. Our statistical mediation analysis suggests that ARDS is the mechanism by which plasma-free 2-ClFA concentrations associate with mortality, focusing attention on the neutrophil-endothelial interaction as a driver of ARDS. Our population was of modest size and all subjects were enrolled from a single center; thus, replication would improve generalizability. The quantification of 2-ClFA was performed using sophisticated mass spectroscopic techniques, and in order for these markers to be tested as a potential enrichment factor or response indicator, a more widely available and facile assay would be necessary. We observed correlation between free plasma 2-ClFA concentrations and markers of endothelial activation; however, the degree of correlation would be statistically described as very weak (rho $<0.2$, for 2-ClPA and E selectin) or weak (rho 0.2-0.3 for Ang-2 and sTM). This degree of correlation is similar to that reported between different vascular markers in a study of comparable size (62) but weaker than the correlation previously reported between Ang-2 and IL-6 (63). Finally, further mechanistic work to understand how 2-ClFA affects endothelial activation, and the degree to which this axis can be manipulated pharmacologically, is needed in order to translate these findings to new ARDS therapies.

In summary, we have used mass spectroscopy to detect markers of neutrophil-mediated injury that may functionally identify the pathologic neutrophil contribution to sepsis-associated ARDS. Circulating levels of 2-chloropalmitic and 2-chlorstearic acid strongly associate with sepsis-associated ARDS and mortality, and these chlorinated lipids incite lung microvascular permeability and upregulation of adhesion molecules that could further promote neutrophil-endothelial interaction. Plasma 2-ClFAs should be investigated as markers of a neutrophil-mediated ARDS endotype that may be amenable to precision therapy. 


\section{Methods}

Patient population. The MESSI cohort study enrolls adult subjects, with strongly suspected or confirmed infection and new organ dysfunction, who are admitted to the medical ICU at the Hospital of Pennsylvania $(42,64,65)$. Informed consent was obtained from patients or their proxies. ARDS was defined in accordance with the Berlin definition, with the added requirement of invasive mechanical ventilation over the first 6 days of ICU admission to be confident that the ARDS was attributable to the first septic episode $(66,67)$; chest radiographs were interpreted as described previously $(42,68)$. Acute kidney injury was determined by Acute Kidney Injury Network criteria (69). Neutropenia was defined as an absolute neutrophil count of less than 1,000/microliter on the day of ICU admission as previously described (42). Mortality was defined at 30 days. Plasma was drawn into a 6-ml citrated vacutainer for clinical coagulation testing on arrival to the Emergency Department or upon transfer to the ICU for patients previously on the floor. Samples were spun (3,000 $\mathrm{g}$ for 15 minutes) upon receipt, kept at $4^{\circ} \mathrm{C}$ for 24 hours, and then aliquoted and frozen at $-80^{\circ} \mathrm{C}$ for research purposes. (42). Plasma cytokines and endothelial markers were assayed by ELISA as described previously (42). Healthy controls $(n=4)$ were voluntary donors responding to a solicitation notice at St. Louis University who provided informed consent. Medical history was not obtained. Plasma was collected as described above and frozen at $-80^{\circ} \mathrm{C}$ until analysis.

Plasma 2-ClFA and MPO analyses. Twenty-five microliters of plasma were used to determine free and total 2-ClFA levels as previously described (70). In brief, $105 \mathrm{fmol}$ of 2-chloro-[ $\left.d_{-}-7,7,8,8\right]$-PA was added during a modified Dole extraction to assess free 2-ClFA. For total 2-ClFA analyses, plasma was first subjected to base-catalyzed hydrolysis prior to Dole extraction. Extracted 2-ClFA was then quantitated using LC/MS with electrospray ionization in the negative ion mode and selected reaction monitoring of individual 2-ClFA molecular species. MPO levels were assessed by using the FDA-cleared in vitro diagnostic assay, CardioMPO (Cleveland HeartLab), on autoanalyzer according to the manufacturer's instructions. For 2-ClFA and MPO analyses, deidentified plasma samples were sent to either DAF (2-ClFA) or SLH (MPO) for analyses, and data for each coded sample were then sent to NJM for data merging into the MESSI data set.

Lung microvascular endothelial cell studies. For permeability studies, HMVEC-L were grown to confluence on Transwell polycarbonate filters $(6.5-\mathrm{mm}$ diameter, $0.4-\mu \mathrm{m}$ pore size) mounted in a chamber insert. Resistance across cells was monitored daily using an epithelial volt/ohm meter (EVOM, World Precision Instruments). Following consistent resistance for 3 consecutive days, experiments were performed. Resistance was measured for each well prior to the addition of $10 \mu \mathrm{M}$ BSA-conjugated lipids and then at selected time points. To assess Ang-2 release, lung microvascular endothelial cells were plated in 16-mm diameter wells and grown to confluence. Cells were treated with $10 \mu \mathrm{M}$ BSA-conjugated lipids in growth media with 5\% FBS for 30 minutes. Supernatant was removed and centrifuged for 2,000 $\mathrm{g}$ for 10 minutes to remove cellular debris. Human angiopoietin-2 Quantikine ELISA (R\&D Systems) was used to quantify Ang-2 in the supernatant. Fifty microliters were assayed following manufacturer's protocol.

Cell surface expression of adhesion molecules was measured after lipid treatment of HMVEC-L. Cells were plated in 16-mm wells and grown to confluence. Cells were treated with $10 \mu \mathrm{M}$ BSA-conjugated lipids in growth media with 5\% FBS for 30 minutes (P selectin), 1 hour (E selectin), or 4 hours (ICAM-1 and VCAM-1). HMVEC-L were fixed with 1\% paraformaldehyde overnight. Cells were washed 3 times with PBS and blocked with PBS containing BSA and fish gelatin for 1 hour. Cells were incubated with primary antibodies (1:50) against P selectin (Santa Cruz, catalog sc-8419, monoclonal), E selectin (Santa Cruz, catalog sc-5262, monoclonal), ICAM-1 (Santa Cruz, catalog sc53336, monoclonal), and VCAM-1 (Santa Cruz, catalog 13160 , monoclonal) for 1 hour at $37^{\circ} \mathrm{C}$. Cells were washed twice with PBS and incubated with HRP-conjugated goat anti-mouse secondary antibody $(1: 5,000)$ for 30 minutes at $37^{\circ} \mathrm{C}$. Cells were washed 4 times with PBS and incubated with the 3,3,5,5,tetramethylbenzidine substrate system for 30 minutes in the dark. The color reaction was stopped with $8 \mathrm{~N} \mathrm{H}_{2} \mathrm{SO}_{4}$, and absorbance was read at $450 \mathrm{~nm}$. Absorbance in the presence of secondary antibody alone was subtracted from each sample absorbance. To assess leukocyte adherence to HMVEC-L, endothelial cells were grown to confluence in 22-mm wells. HMVEC-L were treated with $10 \mu \mathrm{M}$ BSA-conjugated lipids in growth media for 4 hours. Leukocytes were isolated from healthy volunteers $(25,71) .2 \times 10^{6}$ leukocytes were subsequently added to lung microvascular endothelial cells and incubated for 20 minutes. Unbound leukocytes were washed away with 3 PBS rinses. The remaining leukocytes were lysed with $0.2 \%$ Triton X-100 and transferred to an Eppendorf tube. 
Tubes were briefly sonicated to ensure complete lysis of cells. To measure MPO, $400 \mu 1$ cell lysate was transferred to a test tube containing phosphate buffer, Hank's buffer with BSA, 1,9-dimethyl-methylene blue, and $0.5 \%$ hydrogen peroxide. Samples were incubated for 15 minutes at room temperature. Sodium azide (1\%) was added to stop the reaction. Absorbance was measured at $460 \mathrm{~nm}$. Adherence of platelets to lung microvascular endothelial cells was performed as previously described (72). Briefly, HMVEC-L were grown to confluence in 22-mm wells. Cells were treated with $10 \mu \mathrm{M}$ BSA-conjugated lipids in growth media for 4 hours. Platelets were isolated from healthy volunteers (73). Platelets were labeled with Calcein-AM (2.5 $\mu \mathrm{mol} / 1$ ) for 15 minutes at $37^{\circ} \mathrm{C}$ in the dark. Fluorescence-labeled platelets were subsequently added to lung microvascular endothelial cells and incubated for 20 minutes at $37^{\circ} \mathrm{C}$. Unbound platelets were washed away with 3 PBS rinses. The remaining platelets were lysed in lysis buffer for 10 minutes, and fluorescence was measured with a plate reader (excitation at $492 \mathrm{~nm}$, emission at $535 \mathrm{~nm}$ ).

Statistics. Clinical characteristics were compared between ARDS and non-ARDS subjects using Pearson's $\chi^{2}$ test or Fishers exact test for categorical data and 2-tailed Student's $t$ or Wilcoxon rank-sum test as appropriate for continuous data. Plasma 2-ClFA concentrations were compared between clinical groups and between experimental conditions using the Wilcoxon rank-sum test or ANOVA. Because the distribution of 2-CIPA and 2-CLSA were right-skewed, we performed log transformation for normality using Stata 12.1. We performed multivariable logistic regression of ARDS using log-transformed 2-ClFA concentrations and APACHE III score, age, sex, European descent, and pulmonary source of infection, given our prior reported associations in this population (42). For multivariable logistic regression of 30-day mortality, we included covariates APACHE III score, age, sex, absolute neutrophil count, and European descent as covariates $(42,45)$. The lowest absolute neutrophil count on the day of ICU admission was included as a covariate to ensure that associations with 2-ClFA were independent of neutrophil count. Correlation between plasma markers was evaluated with Spearman rank correlation. We employed mediation analysis as a formal approach to explain the mechanism by which an explanatory variable (2-ClFA) influences the outcome (mortality) via a third mediator variable (ARDS) (74). We used logistic regression to estimate the change in ARDS risk for each log change in free 2-ClFA, which we term the mediator effect, and logistic regression of 2-CIFA, ARDS, an interaction term [ARDS $\times$ $\log$ (free 2-ClFA)], APACHE III score, and age to model the total effect of 2-ClFA on mortality, as enacted in $\mathrm{R}$ statistical packages. To assess whether plasma 2-ClFA concentrations had predictive utility for ARDS compared with the APACHE III severity of illness score $(42,45)$, we compared AUROC by $\chi^{2}$ test for models predicting ARDS based on APACHE III alone or in combination with log-transformed free 2-ClFA levels $(75,76)$. Using methodology described by Pencina et al. and enacted in Stata, we calculated the ARDS net reclassification index and the IDI for models that combined APACHE III and plasma 2-ClFA concentration (dichotomized at the median 2-ClFA level) compared to the base model of APACHE III score alone $(77,78)$. We used the nonparametric test of trend to assess for an association between sepsis severity and 2-ClFA, considering subjects in a stepwise fashion from healthy controls to sepsis survivors to sepsis nonsurvivors. For in vitro experiments, endothelial barrier function was compared among control, PA, and 2-chloropalmitic acid treatment by ANOVA with Tukey's post-hoc test at individual time points. $P$ values of less than 0.05 were considered significant.

Study approval. Leukocytes and platelets were isolated from healthy volunteers, as approved by Saint Louis University Institutional Review Board protocols 9952 and 12369, respectively. MESSI samples were collected as approved by University of Pennsylvania Institutional Review Board protocol 808542.

\section{Author contributions}

NJM contributed to study conception, experimental design, clinical study data collection, statistical analyses, and wrote the manuscript. JPR contributed to study conception, experimental design, clinical study data collection, statistical analyses, and wrote the manuscript. RF performed statistical analyses and wrote the manuscript. JDC contributed to study conception, experimental design, and wrote the manuscript. SLH contributed to experimental design, plasma MPO studies, and wrote the manuscript. CLH performed endothelial studies and contributed to experimental design. CJA and JDF performed 2-ClFA analyses and contributed to experimental design. JM performed endothelial studies, contributed to study conception, and contributed to experimental design. DAF contributed to study conception, experimental design, 2-ClFA analyses, and wrote the manuscript. 


\section{Acknowledgments}

This study was supported by NIH grants R01GM115553 (to DAF and JM), R01HL122474 (to NJM), and P01HL076491 (to SLH).

Address correspondence to: David A. Ford, Saint Louis University School of Medicine, Department of Biochemistry and Molecular Biology, 1100 South Grand Ave., Doisy Research Center, Saint Louis, Missouri 63104, USA. Phone: 314.977.9264; Email: fordda@slu.edu.

1. Singer M, et al. The Third International Consensus Definitions for Sepsis and Septic Shock (Sepsis-3). JAMA. 2016;315(8):801-810.

2. Brown KA, Brain SD, Pearson JD, Edgeworth JD, Lewis SM, Treacher DF. Neutrophils in development of multiple organ failure in sepsis. Lancet. 2006;368(9530):157-169.

3. Cepinskas G, Wilson JX. Inflammatory response in microvascular endothelium in sepsis: role of oxidants. J Clin Biochem Nutr. 2008;42(3):175-184

4. Aziz M, Jacob A, Yang WL, Matsuda A, Wang P. Current trends in inflammatory and immunomodulatory mediators in sepsis. J Leukoc Biol. 2013;93(3):329-342.

5. Spite M, et al. Resolvin D2 is a potent regulator of leukocytes and controls microbial sepsis. Nature. 2009;461(7268):1287-1291

6. Teixeira-da-Cunha MG, et al. Bacterial clearance is improved in septic mice by platelet-activating factor-acetylhydrolase (PAF-AH) administration. PLoS One. 2013;8(9):e74567.

7. Ware LB, Fessel JP, May AK, Roberts LJ. Plasma biomarkers of oxidant stress and development of organ failure in severe sepsis. Shock. 2011;36(1):12-17.

8. Gräler MH. The role of sphingosine 1-phosphate in immunity and sepsis. Am J Clin Exp Immunol. 2012;1(2):90-100.

9. Fang WF, et al. Lipid A fraction of LPS induces a discrete MAPK activation in acute lung injury. Am J Physiol Lung Cell Mol Physiol. 2007;293(2):L336-L344.

10. [No authors listed]. Randomized, placebo-controlled trial of lisofylline for early treatment of acute lung injury and acute respiratory distress syndrome. Crit Care Med. 2002;30(1):1-6.

11. Bellani G, et al. Epidemiology, patterns of care, and mortality for patients with acute respiratory distress syndrome in intensive care units in 50 countries. JAMA. 2016;315(8):788-800

12. Gaut JP, et al. Neutrophils employ the myeloperoxidase system to generate antimicrobial brominating and chlorinating oxidants during sepsis. Proc Natl Acad Sci U S A. 2001;98(21):11961-11966.

13. Klebanoff SJ. Oxygen metabolism and the toxic properties of phagocytes. Ann Intern Med. 1980;93(3):480-489.

14. Nathan C. Neutrophils and immunity: challenges and opportunities. Nat Rev Immunol. 2006;6(3):173-182.

15. Adams JM, Hauser CJ, Livingston DH, Lavery RF, Fekete Z, Deitch EA. Early trauma polymorphonuclear neutrophil responses to chemokines are associated with development of sepsis, pneumonia, and organ failure. J Trauma. 2001;51(3):452-456.

16. Brealey D, Singer M. Multi-organ dysfunction in the critically ill: effects on different organs. JR Coll Physicians Lond. 2000;34(5):428-431.

17. Nuytinck HK, Offermans XJ, Kubat K, Goris JA. Whole-body inflammation in trauma patients. An autopsy study. Arch Surg. 1988;123(12):1519-1524.

18. Thijs A, Thijs LG. Pathogenesis of renal failure in sepsis. Kidney Int Suppl. 1998;66:S34-S37.

19. Windsor AC, Mullen PG, Fowler AA, Sugerman HJ. Role of the neutrophil in adult respiratory distress syndrome. Br J Surg. 1993;80(1):10-17.

20. Kangelaris KN, et al. Increased expression of neutrophil-related genes in patients with early sepsis-induced ARDS. Am J Physiol Lung Cell Mol Physiol. 2015;308(11):L1102-L1113.

21. Weiss SJ. Tissue destruction by neutrophils. N Engl J Med. 1989;320(6):365-376.

22. Kothari N, et al. Increased myeloperoxidase enzyme activity in plasma is an indicator of inflammation and onset of sepsis. J Crit Care. 2011;26(4):435.e1-435.e7.

23. Harrison JE, Schultz J. Studies on the chlorinating activity of myeloperoxidase. J Biol Chem. 1976;251(5):1371-1374.

24. Albert CJ, Crowley JR, Hsu FF, Thukkani AK, Ford DA. Reactive chlorinating species produced by myeloperoxidase target the vinyl ether bond of plasmalogens: identification of 2-chlorohexadecanal. J Biol Chem. 2001;276(26):23733-23741.

25. Thukkani AK, Hsu FF, Crowley JR, Wysolmerski RB, Albert CJ, Ford DA. Reactive chlorinating species produced during neutrophil activation target tissue plasmalogens: production of the chemoattractant, 2-chlorohexadecanal. J Biol Chem. 2002;277(6):3842-3849.

26. Thukkani AK, et al. Myeloperoxidase-derived reactive chlorinating species from human monocytes target plasmalogens in low density lipoprotein. J Biol Chem. 2003;278(38):36365-36372.

27. Chilton FH, Connell TR. 1-ether-linked phosphoglycerides. Major endogenous sources of arachidonate in the human neutrophil. J Biol Chem. 1988;263(11):5260-5265.

28. Ford DA, Gross RW. Plasmenylethanolamine is the major storage depot for arachidonic acid in rabbit vascular smooth muscle and is rapidly hydrolyzed after angiotensin II stimulation. Proc Natl Acad Sci U S A. 1989;86(10):3479-3483.

29. Hsu FF, Turk J, Thukkani AK, Messner MC, Wildsmith KR, Ford DA. Characterization of alkylacyl, alk-1-enylacyl and lyso subclasses of glycerophosphocholine by tandem quadrupole mass spectrometry with electrospray ionization. J Mass Spectrom. 2003;38(7):752-763.

30. McHowat J, Jones JH, Creer MH. Gradient elution reversed-phase chromatographic isolation of individual glycerophospholipid molecular species. J Chromatogr B Biomed Sci Appl. 1997;702(1-2):21-32.

31. Murphy EJ, Joseph L, Stephens R, Horrocks LA. Phospholipid composition of cultured human endothelial cells. Lipids. 
1992;27(2):150-153.

32. Hazen SL, Hall CR, Ford DA, Gross RW. Isolation of a human myocardial cytosolic phospholipase A2 isoform. Fast atom bombardment mass spectroscopic and reverse-phase high pressure liquid chromatography identification of choline and ethanolamine glycerophospholipid substrates. J Clin Invest. 1993;91(6):2513-2522.

33. Dorman RV, Dreyfus H, Freysz L, Horrocks LA. Ether lipid content of phosphoglycerides from the retina and brain of chicken and calf. Biochim Biophys Acta. 1976;486(1):55-59.

34. Portilla D, Creer MH. Plasmalogen phospholipid hydrolysis during hypoxic injury of rabbit proximal tubules. Kidney Int. 1995;47(4):1087-1094

35. Anbukumar DS, et al. Chlorinated lipid species in activated human neutrophils: lipid metabolites of 2-chlorohexadecanal. J Lipid Res. 2010;51(5):1085-1092.

36. Brahmbhatt VV, Albert CJ, Anbukumar DS, Cunningham BA, Neumann WL, Ford DA. \{Omega\}-oxidation of \{alpha\}-chlorinated fatty acids: identification of \{alpha\}-chlorinated dicarboxylic acids. J Biol Chem. 2010;285(53):41255-41269.

37. Wildsmith KR, Albert CJ, Anbukumar DS, Ford DA. Metabolism of myeloperoxidase-derived 2-chlorohexadecanal. J Biol Chem. 2006;281(25):16849-16860.

38. Messner MC, Albert CJ, Ford DA. 2-Chlorohexadecanal and 2-chlorohexadecanoic acid induce COX-2 expression in human coronary artery endothelial cells. Lipids. 2008;43(7):581-588.

39. Thukkani AK, Martinson BD, Albert CJ, Vogler GA, Ford DA. Neutrophil-mediated accumulation of 2-ClHDA during myocardial infarction: 2-ClHDA-mediated myocardial injury. Am J Physiol Heart Circ Physiol. 2005;288(6):H2955-H2964.

40. Wang WY, Albert CJ, Ford DA. Alpha-chlorofatty acid accumulates in activated monocytes and causes apoptosis through reactive oxygen species production and endoplasmic reticulum stress. Arterioscler Thromb Vasc Biol. 2014;34(3):526-532.

41. Grommes J, Soehnlein O. Contribution of neutrophils to acute lung injury. Mol Med. 2011;17(3-4):293-307.

42. Reilly JP, et al. Neutropenic sepsis is associated with distinct clinical and biological characteristics: a cohort study of severe sepsis. Crit Care. 2016;20(1):222.

43. Braverman NE, Moser AB. Functions of plasmalogen lipids in health and disease. Biochim Biophys Acta. 2012;1822(9):1442-1452.

44. Ford DA. Lipid oxidation by hypochlorous acid: chlorinated lipids in atherosclerosis and myocardial ischemia. Clin Lipidol. 2010;5(6):835-852.

45. Knaus WA, et al. The APACHE III prognostic system. Risk prediction of hospital mortality for critically ill hospitalized adults. Chest. 1991;100(6):1619-1636.

46. Fiedler U, et al. The Tie-2 ligand angiopoietin-2 is stored in and rapidly released upon stimulation from endothelial cell Weibel-Palade bodies. Blood. 2004;103(11):4150-4156.

47. Ford DA, et al. Formation of chlorinated lipids post-chlorine gas exposure. J Lipid Res. 2016;57(8):1529-1540.

48. Kothari N, et al. Increased myeloperoxidase enzyme activity in plasma is an indicator of inflammation and onset of sepsis J Crit Care. 2011;26(4):435.e1-435.e7.

49. Parikh SM, et al. Excess circulating angiopoietin-2 may contribute to pulmonary vascular leak in sepsis in humans. PLoS Med. 2006;3(3):e46

50. Pittet JF, Mackersie RC, Martin TR, Matthay MA. Biological markers of acute lung injury: prognostic and pathogenetic significance. Am J Respir Crit Care Med. 1997;155(4):1187-1205.

51. Ranieri VM, et al. Effect of mechanical ventilation on inflammatory mediators in patients with acute respiratory distress syndrome: a randomized controlled trial. JAMA. 1999;282(1):54-61.

52. Parsons PE, et al. Lower tidal volume ventilation and plasma cytokine markers of inflammation in patients with acute lung injury. Crit Care Med. 2005;33(1):1-6.

53. Suratt BT, et al. Plasma granulocyte colony-stimulating factor levels correlate with clinical outcomes in patients with acute lung injury. Crit Care Med. 2009;37(4):1322-1328.

54. Suwa T, Hogg JC, Klut ME, Hards J, van Eeden SF. Interleukin-6 changes deformability of neutrophils and induces their sequestration in the lung. Am J Respir Crit Care Med. 2001;163(4):970-976.

55. Mokart D, et al. Monocyte deactivation in neutropenic acute respiratory distress syndrome patients treated with granulocyte colony-stimulating factor. Crit Care. 2008;12(1):R17.

56. Prescott SM, McIntyre TM, Zimmerman G. Two of the usual suspects, platelet-activating factor and its receptor, implicated in acute lung injury. J Clin Invest. 1999;104(8):1019-1020.

57. Famous KR, et al. Acute respiratory distress syndrome subphenotypes respond differently to randomized fluid management strategy. Am J Respir Crit Care Med. 2017;195(3):331-338.

58. Calfee CS, et al. Subphenotypes in acute respiratory distress syndrome: latent class analysis of data from two randomised controlled trials. Lancet Respir Med. 2014;2(8):611-620.

59. Iwata K, et al. Effect of neutrophil elastase inhibitor (sivelestat sodium) in the treatment of acute lung injury (ALI) and acute respiratory distress syndrome (ARDS): a systematic review and meta-analysis. Intern Med. 2010;49(22):2423-2432.

60. Zeiher BG, et al. Neutrophil elastase inhibition in acute lung injury: results of the STRIVE study. Crit Care Med. 2004;32(8):1695-1702.

61. David S, et al. Angiopoietin-2 may contribute to multiple organ dysfunction and death in sepsis*. Crit Care Med 2012;40(11):3034-3041

62. Shapiro NI, et al. The association of endothelial cell signaling, severity of illness, and organ dysfunction in sepsis. Crit Care. 2010;14(5):R182.

63. Robinson-Cohen C, et al. Association of markers of endothelial dysregulation Ang1 and Ang2 with acute kidney injury in critically ill patients. Crit Care. 2016;20(1):207.

64. Anderson BJ, et al. Admission plasma levels of the neuronal injury marker neuron-specific enolase are associated with mortality and delirium in sepsis. J Crit Care. 2016;36:18-23.

65. Reilly JP, et al. ABO blood type A is associated with increased risk of ARDS in whites following both major trauma and severe sepsis. Chest. 2014;145(4):753-761.

66. ARDS Definition Task Force, et al. Acute respiratory distress syndrome: the Berlin definition. JAMA. 2012;307(23):2526-2533. 
67. Gajic O, et al. Early identification of patients at risk of acute lung injury: evaluation of lung injury prediction score in a multicenter cohort study. Am J Respir Crit Care Med. 2011;183(4):462-470.

68. Shah $\mathrm{CV}$, et al. An alternative method of acute lung injury classification for use in observational studies. Chest. 2010;138(5):1054-1061.

69. Reilly JP, et al. The ABO histo-blood group and AKI in critically ill patients with trauma or sepsis. Clin J Am Soc Nephrol. 2015;10(11):1911-1920.

70. Wacker BK, Albert CJ, Ford BA, Ford DA. Strategies for the analysis of chlorinated lipids in biological systems. Free Radic Biol Med. 2013;59:92-99.

71. Ferrante A, Thong YH. Optimal conditions for simultaneous purification of mononuclear and polymorphonuclear leucocytes from human blood by the Hypaque-Ficoll method. J Immunol Methods. 1980;36(2):109-117.

72. Verheul HM, Jorna AS, Hoekman K, Broxterman HJ, Gebbink MF, Pinedo HM. Vascular endothelial growth factor-stimulated endothelial cells promote adhesion and activation of platelets. Blood. 2000;96(13):4216-4221.

73. Beckett CS, Kell PJ, Creer MH, McHowat J. Phospholipase A2-catalyzed hydrolysis of plasmalogen phospholipids in thrombin-stimulated human platelets. Thromb Res. 2007;120(2):259-268.

74. Imai K, Keele L, Tingley D. A general approach to causal mediation analysis. Psychol Methods. 2010;15(4):309-334.

75. Pepe MS, Cai T, Longton G. Combining predictors for classification using the area under the receiver operating characteristic curve. Biometrics. 2006;62(1):221-229.

76. Pepe PE, Potkin RT, Reus DH, Hudson LD, Carrico CJ. Clinical predictors of the adult respiratory distress syndrome. Am $J$ Surg. 1982;144(1):124-130.

77. Pencina MJ, D'Agostino RB Sr, D'Agostino RB Jr, Vasan RS. Evaluating the added predictive ability of a new marker: from area under the ROC curve to reclassification and beyond. Stat Med. 2008;27(2):157-172.

78. Steyerberg EW, et al. Assessing the performance of prediction models: a framework for traditional and novel measures. Epidemi ology. 2010;21(1):128-138. 